\title{
Thyroid Dysfunction in Patients with Systemic Lupus Erythematosus, Correlation with Disease Activity
}

\author{
M. Salah Abd El-Baky ${ }^{1}$, Dalia Abdel Mohsen ${ }^{1}$, Dalia F. Mohammed ${ }^{1}$, \\ Hyiada S. Mansour ${ }^{1}$, Noran O. El-Azizi ${ }^{1}$, W. Mohammed $^{2}$, M. Sabry ${ }^{3}$ \\ Departments of Internal Medicine - Rheumatology Division ${ }^{1}$, Radiology ${ }^{2}$, \\ Clinical Pathology ${ }^{3}$, Ain Shams University; Egypt
}

\begin{abstract}
Background: The relationship between autoimmune thyroid disease and systemic lupus erythematosus (SLE) has been revealed but the prevalence of thyroid disease in lupus patients is controversial. The aim of this study is to assess thyroid dysfunction and the presence of anti-thyroid antibodies in patients with SLE, and its association with disease characteristics and disease activity. Methods: Sixty patients with SLE $\geq 18$ years who satisfied the American College of Rheumatology (ACR) criteria and thirty age and sex matched normal volunteer were included, all underwent laboratory evaluation for serum free T3, free T4, TSH, Antithyroglobulin antibody (Ab TG) and Antithyroid peroxidase antibody (Ab TPO). Clinical and serological characteristics and disease activity of SLE were assessed; correlation with thyroid dysfunction was studied. Results: 2(3.33\%) patients had subclinical hyperthyroidism, $24(40 \%)$ were euthyroid, $12(21.67 \%)$ had subclinical hypothyroidism and $22(35 \%)$ had overt hypothyroidism. All the control group was euthyroid. The patients with thyroid dysfunction had more arthralgias, arthritis, changes of voice, bowel habits and weight, irregular menstruation, sleep disturbance, nervousness and tremors than the euthyroid lupus patients $(\mathrm{p}<0.05)$. The lupus patients with subclinical and overt hypothyroidism had statistically significant higher Ab TG than the euthyroid patients and patients with subclinical hyperthyroidism and control group $(\mathrm{p}<0.05)$ but no statistically significant difference between all groups as regard Ab TPO. SLE patients with subclinical \& overt hypothyroidism had statistically significant higher ESR and SLAM score than the euthyroid patients and patients with subclinical hyperthyroidism $(\mathrm{p}<0.05)$. There was a positive correlation between Ab TG levels and body mass index BMI, ESR and disease activity measures by SLAM score $(p<0.05)$. Conclusion: The thyroid dysfunction is more frequent in SLE patients than control group. Subclinical and overt hypothyroidism are more likely to occur in SLE patients. There are a positive correlation between Ab TG level and disease activity and negative correlation with free T3 \& free T4. [Egypt J Rheumatology \& Clinical Immunology, 2014; 2(1): 37-43]
\end{abstract}

Key Words: thyroid dysfunction, systemic lupus erythematosus.

\section{INTRODUCTION}

Systemic lupus erythematosus (SLE) is a prototype of autoimmune diseases affecting predominantly women. It is characterized by a multisystem organ involvement because of dysregulation of selfreactive B cells leading to autoantibody production, immune complex deposition and complement activation with tissue damage ${ }^{1}$. It is important for physicians to be aware of the functional and social consequences of the disease and to improve the patient's quality of life. When treating a patient with SLE, the aim is to suppress disease activity, which is reversible and to prevent the onset of organ damage, which is irreversible. This is facilitated by regular care of SLE and related conditions ${ }^{2}$.

Correspondence: N. O. El-Azizi, Department of Internal Medicine, Ain Shams University; Egypt.

e-mail: nowara2005@yahoo.com
Autoimmune thyroid disease, marked by the presence of antibodies directed against thyroid antigens, has been associated with a number of nonorgan-specific rheumatological disorders ${ }^{3}$.

Although the relationship between autoimmune thyroid disease and SLE has been revealed, the prevalence of thyroid disease in lupus patients is controversial. Reported prevalence of autoimmune thyroid disease and antithyroid antibodies in SLE patients varied considerably ${ }^{4}$.

For this reason the aim of our study was to assess thyroid dysfunction in Egyptian patients with SLE, and its association with disease characteristics and disease activity. 


\section{PATIENTS AND METHODS}

\section{Patients}

Sixty patients with SLE (55 females and 5 males) with ages ranging from 18 to 50 years were recruited from a pool of patients with SLE at the outpatient rheumatology clinic of Ain Shams university hospital, Egypt. All patients were subjected to full medical, general and rheumatological examination. All patients were fulfilling the American College of Rheumatology (ACR) criteria ${ }^{5}$. Rheumatologic evaluation was completed using the Systemic Lupus Activity Measure (SLAM) ${ }^{6}$. Routine Laboratory Investigations (such as CBC, ESR, Kidney, liver functions, complete urine analysis, 24 hours urinary proteins and corrected creatinine clearance), thyroid profile (free T3, free T4 \& TSH) Serological Investigations (ANA, Anti-DNA, Antithyroglobulin antibody $\{\mathrm{Ab} \mathrm{Tg}\}$ and Antithyroid peroxidase antibody $\{\mathrm{Ab} \mathrm{TPO}\})$ was done. Thyroid ultrasonography was also done to assess the thyroid size and the presence of cysts or nodules. SLE patients were stratified according to SLAM scores into two groups active group (SLAM score $>6$ ) and remission group (SLAM score $<6)^{7}$. SLAM measures the current status of SLE disease activity according to clinical and laboratory manifestations. SLE disease activity is defined as the reversible manifestations of the underlying inflammatory process ${ }^{6}$.

\section{Statistical Analysis}

The computer software package SPSS for Windows (Version 17) was used for the data analysis. Continuous variables such as age were expressed as mean \pm standard deviation, whereas categorical variables such as gender were presented as frequencies (\%).

\section{RESULTS}

This study included three groups: Group I active SLE (SLAM score $\geq 6$ ) $\}$ they were 27 females, 3 males and with mean age $27.4 \pm 7.113$ years, Group II \{inactive SLE (SLAM score $<6$ ) $\}$ they were 28 females, 2 males and with mean age $30.117 \pm 8.919$ years) and Group III age, and sex matched controls they were 22 females, 8 males and with a mean age $25.7 \pm 7.207$ years with no significant differences. There were no significant differences as regard the duration of illness and treatment between active and inactive group; as the duration of illness for the active group was $4.954 \pm 3.624$ years, versus $7.561 \pm 4.815$ years in inactive group, meanwhile the mean duration of treatment for the active group was $4.082 \pm 3.715$ years versus $6.803 \pm 4.601$ years in inactive group, while there was highly significant difference as regard SLAM score between both groups $(\mathrm{p}<0.001)$. Medications at the time of the study included prednisone in all cases and disease-modifying anti-rheumatic drugs
(Hydroxycholoroquine and Azathioprine or Cyclophosphamide or Mycophenolate Mofetil).

$2(3.33 \%)$ patients had subclinical hyperthyroidism, 24 (40\%) were euthyroid, 12 $(21.67 \%)$ had subclinical hypothyroidism and 22 $(35 \%)$ had overt hypothyroidism but all the control group were euthyroid. $36(60 \%)$ of SLE patients had thyroid dysfunction; group I had more thyroid dysfunction than group II. In group I (active SLE) 2 (6.67\%) patients had subclinical hyperthyroidism, 3 (10\%) were euthyroid, 7 (23.33\%) had subclinical hypothyroidism, $18(60 \%)$ had overt hypothyroidism and in group II (Inactive SLE) no patients had subclinical hyperthyroidism, 21 (70\%) were euthyroid, $5(16.67 \%)$ had subclinical hypothyroidism, 4 (13.3\%) had overt hypothyroidism (Figure 1).

There were highly significant elevated TSH \& Anti-TG in group I (active SLE) than group II (inactive SLE) than in group III (control) $(\mathrm{p}<0.01)$. Also there was significantly lower Free T4 in group I (active SLE) than in group III (Control) $(\mathrm{p}<0.05)$. Otherwise no significant difference between the 3 groups as regards Free T3 \& Anti-TPO $(\mathrm{P}>0.05)$. No abnormality was detected in thyroid profile of the control group (Table 1).

The SLE patients further subdivided according to thyroid function tests into (subclinical hyperthyroidism, euthyroid, subclinical \& overt hypothyroidism) and by comparing the 4 groups we found that the females were likely to have thyroid dysfunction than males. Subclinical hyperthyroidism had [2 (3.3\%) females \& no males], Subclinical hypothyroidism had [9 (21\%) females \& 3 (5\%) males] and overt hypothyroidism had [21 (35\%) females \& 1 (1.75) male]. There was highly significant difference between the 3 groups as regard SLAM score $(\mathrm{p}<0.01)$; it is higher in the overt hypothyroidism group then subclinical hypothyroidism group than euthyroid group. According to thyroid profile, antibodies and different laboratory investigations there was significant difference between the 4 groups as regard free $\mathrm{T} 4$ $(\mathrm{p}<0.01), \mathrm{TSH}$, Anti-TG, ESR \& serum creatinine $(\mathrm{p}<$ $0.05)$. Otherwise, no significant differences between the 4 groups as regard free T3, Anti-TPO and other laboratory investigations $(\mathrm{P}>0.05)$. Also we found no statistical significant difference between 4 groups as regard age of patients and disease duration.

The patients with thyroid dysfunction group had complaining more from arthralgia/arthritis, hoarseness of voice, change in appetite, bowel habits, nervousness, weight, duration \& amount of menstruation, sleep disturbance $\&$ puffiness in the eye and on examination more number of patients had fine tremors, pallor, cold hand, hair loss \& thyroid enlargement than the euthyroid group but without statistically significant difference. 
Abnormal finding in thyroid U/S was found in; 1 (1.7\%) patient in the subclinical hyperthyroidism group [had left thyroid cyst], $5(8.3 \%)$ patients in euthyroid group [ 2 patients had left \& 1 patient right thyroid cyst, 1 patient had diffuse multinodular goiter \& 1 patient had surgically resected left thyroid lobe] and $1(1.7 \%)$ patient in hypothyroid group [had diffuse multinodular goiter].

There was a significant positive correlation between Ab TG level \& Body Mass Index (BMI), ESR, SLAM score and significant negative correlation with free $\mathrm{T} 3$ and free $\mathrm{T} 4(\mathrm{P}<0.05)$ (Figure 2).

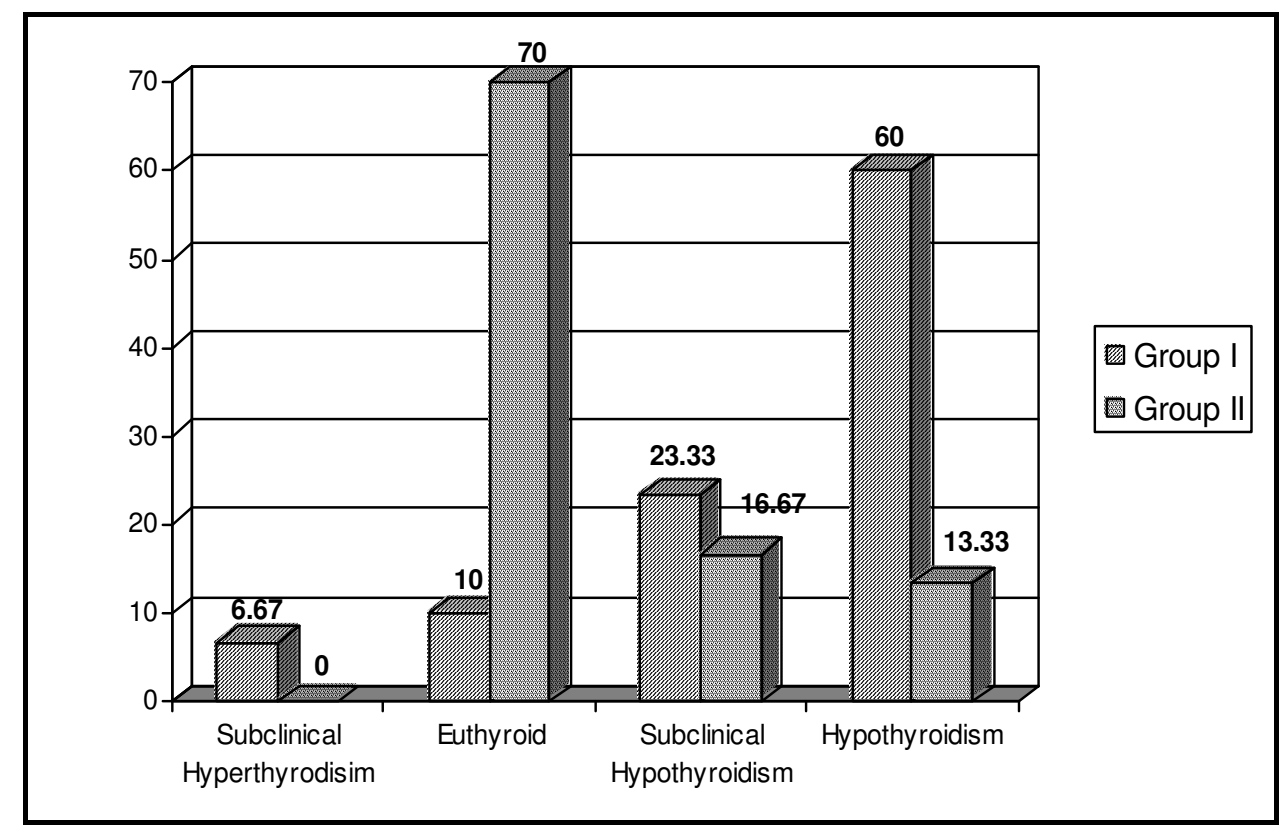

Figure 1. Classification of the SLE patients in Group I (Active SLE) \& II (Inactive SLE) according to thyroid functions.

Table 1. Comparative study between Group I (Active SLE), II (Inactive SLE) \& III (Control) as a regard thyroid function tests \& antithyroid antibodies.

\begin{tabular}{|c|c|c|c|c|c|c|c|}
\hline \multicolumn{2}{|c|}{$\begin{array}{c}\text { Thyroid Function Tests \& } \\
\text { Antibodies }\end{array}$} & \multirow{2}{*}{$\begin{array}{c}\text { Range } \\
0.20-3.00\end{array}$} & \multirow{2}{*}{$\begin{array}{c}\text { Mean } \pm \text { SD } \\
1.912 \pm 0.549\end{array}$} & \multirow{2}{*}{$\begin{array}{c}\text { ANOVA } \\
\text { P-Value } \\
\text { \& Sig } \\
\end{array}$} & \multicolumn{2}{|c|}{ TUKEY'S Test } & \multirow{2}{*}{$\begin{array}{l}\text { Sig } \\
\text { NS }\end{array}$} \\
\hline \multirow{3}{*}{$\begin{array}{l}\text { Free T3 } \\
(1.4-4.2)\end{array}$} & Group I (Active SLE) & & & & I\&II & 0.279 & \\
\hline & Group II (Inactive SLE) & $1.60-3.25$ & $2.125 \pm 0.457$ & \multirow{2}{*}{$\begin{array}{l}0.131 \\
\text { NS }\end{array}$} & I\&III & 0.917 & NS \\
\hline & Group III (Control) & $1.05-4.00$ & $2.18 \pm 0.598$ & & II\&III & 0.136 & NS \\
\hline \multirow{3}{*}{$\begin{array}{l}\text { Free T4 } \\
(0.8-2.4)\end{array}$} & Group I (Active SLE) & $0.10-1.92$ & $0.815 \pm 0.386$ & \multirow{3}{*}{$\begin{array}{c}0.001 \\
\mathrm{HS}\end{array}$} & I\&II & 0.102 & NS \\
\hline & Group II (Inactive SLE) & $0.54-1.80$ & $0.991 \pm 0.356$ & & I\&III & 0.000 & S \\
\hline & Group III (Control) & $0.79-1.66$ & $1.154 \pm 0.222$ & & II\&III & 0.139 & NS \\
\hline \multirow{3}{*}{$\begin{array}{c}\text { TSH } \\
(0.5-5.6)\end{array}$} & Group I (Active SLE) & $0.10-40.00$ & $7.685 \pm 10.172$ & \multirow{3}{*}{$\begin{array}{l}0.014 \\
S\end{array}$} & I\&II & 0.036 & $\mathrm{~S}$ \\
\hline & Group II (Inactive SLE) & $0.50-8.20$ & $3.776 \pm 1.979$ & & I\&III & 0.986 & NS \\
\hline & Group III (Control) & $0.55-5.60$ & $3.532 \pm 1.175$ & & II\&III & 0.024 & $\mathrm{~S}$ \\
\hline \multirow{3}{*}{$\begin{array}{l}\text { Anti-TPO } \\
(<50)\end{array}$} & Group I (Active SLE) & $12.00-145.00$ & $28.90 \pm 30.008$ & \multirow{3}{*}{$\begin{array}{l}0.096 \\
\text { NS }\end{array}$} & I\&II & 0.158 & NS \\
\hline & Group II (Inactive SLE) & $10.00-42.00$ & $20.133 \pm 6.725$ & & I\&III & 0.996 & NS \\
\hline & Group III (Control) & $10.00-42.00$ & $19.733 \pm 7.647$ & & II\&III & 0.134 & NS \\
\hline \multirow{3}{*}{$\begin{array}{l}\text { Anti-TG } \\
(<100)\end{array}$} & Group I (Active SLE) & $23.00-500.00$ & $85.767 \pm 93.821$ & \multirow{3}{*}{$\begin{array}{c}0.001 \\
\text { HS }\end{array}$} & I\&II & 0.004 & $S$ \\
\hline & Group II (Inactive SLE) & $25.00-100.00$ & $38.20 \pm 16.52$ & & I\&III & 0.003 & S \\
\hline & Group III (Control) & $17.00-85.00$ & $36.867 \pm 12.099$ & & II\&III & 0.995 & NS \\
\hline
\end{tabular}



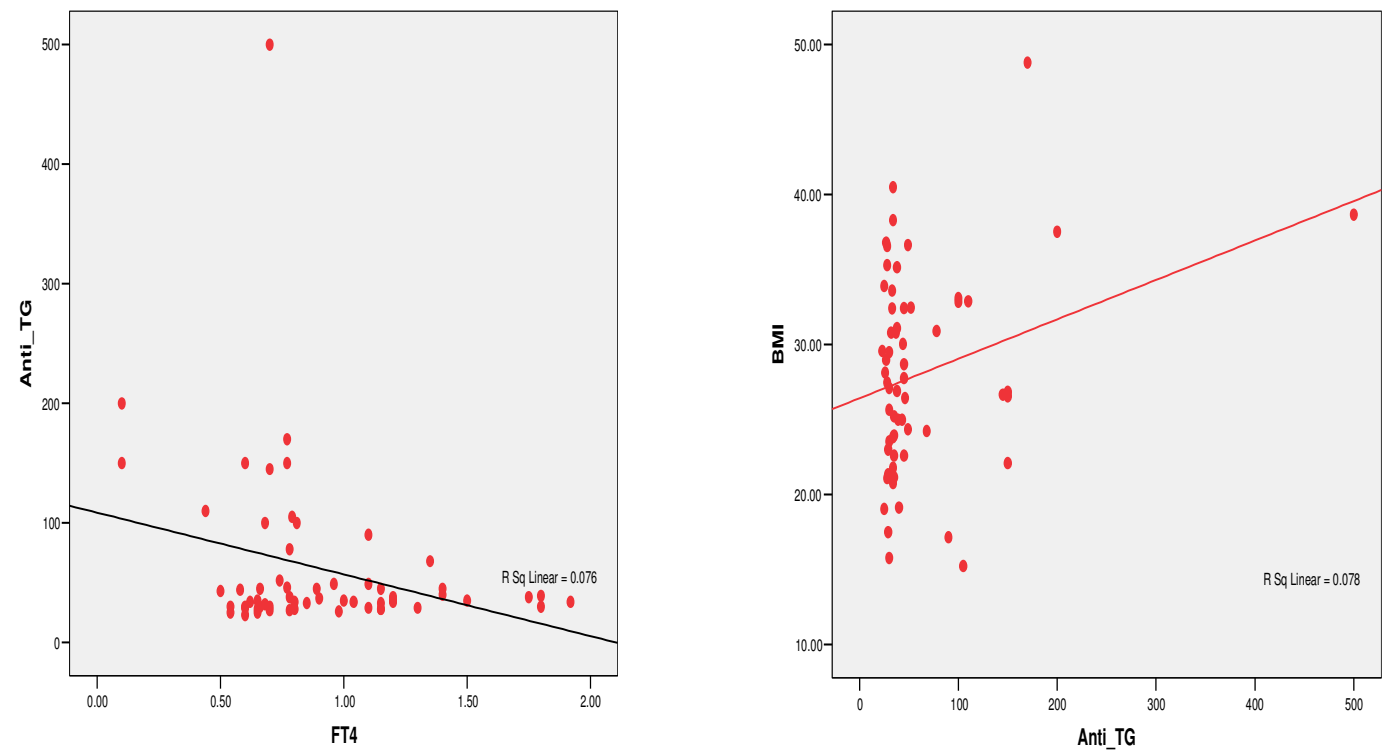

Figure 2. Correlation between thyroid antibodies \& different laboratory, measurements among SLE patients.

\section{DISCUSSION}

Our results showed that $36(60 \%)$ of SLE patients had thyroid dysfunction; 27 (90\%) patients in group I (active SLE) and 9 (30\%) patients in group II (inactive SLE) and no thyroid dysfunction was found in control group. The most common thyroid dysfunction was overt hypothyroidism 22 (35\%) patients then subclinical hypothyroidism $12(21.67 \%)$ patients then finally subclinical hyperthyroidism 2 $(3.33 \%)$ patients from this data we concluded that group I (active SLE) patients had more thyroid dysfunction than group II (inactive SLE) patients. Many studies tried to find the prevalence of thyroid dysfunction among the SLE patients \& the prevalence of antithyroid antibodies among different populations. Our results agreed with many studies; as Miller et al. ${ }^{8}$, found that in 332 SLE patients hospitalized during a 5year period, the overall prevalence of diagnosed thyroid disease $7.5 \%$ was similar to that in other female populations, but the prevalence of diagnosed hypothyroidism $6.6 \%$ was unexpectedly high. There was also a high frequency of abnormal thyroid function test results in $175(52.7 \%)$ SLE patients without diagnosed thyroid disease who underwent laboratory screening. Weetman \& Walport $^{9}$ in UK reported that prevalence of hypothyroidism in SLE as $24 \%$. However, these patients were clinically euthyroid, although biochemically hypothyroid as defined by a raised TSH. Also Chan et al. ${ }^{10}$ has shown that $13 \%$ of SLE patients had subclinical hypothyroidism. In Egypt, El-Sharif et al. ${ }^{11}$ revealed that thyroid disorders in SLE patients were 50\%, $20 \%$ had euthyroid sick syndrome, $20 \%$ had hypothyroidism (10\% subclinical and $10 \%$ overt), and $10 \%$ had hyperthyroidism (5\% subclinical and 5\% overt). Zakeri. \& Sandooghi ${ }^{12}$ on Southeast Iran SLE patients reported that $24.1 \%$ of SLE patients had thyroid dysfunction. Also Antonelli et al. ${ }^{13}$, found that higher prevalence of clinical hypothyroidism in female SLE patients than in controls and no cases of clinical hyperthyroidism. Also Kumar et al. ${ }^{14}$, found that $36 \%$ of lupus patients had thyroid dysfunction. Primary hypothyroidism was the commonest dysfunction in $14 \%$, while subclinical hypothyroidism and subclinical hyperthyroidism was seen in $12 \%$ and $2 \%$, respectively. Porkodi et al. ${ }^{15}$, found that 20 out of 153 SLE patients $13.1 \%$ had thyroid dysfunction which was less than our incidence. But he agrees with us that clinical hypothyroid was the commonest dysfunction in $60 \%$ of patients, subclinical hypothyroid (TSH alone raised) was the next dysfunction seen in $20 \%$ of patients and hyperthyroidism, a less common abnormality occurred in $10 \%$. But our results was discordant with other studies as Vianna et al. ${ }^{16}$, Tsai et al. ${ }^{17}$ on chinese and Boey et al. ${ }^{18}$ on SLE patients in Singaphora, studies, Chan et al. ${ }^{10}$, Pyne \& Isenberg ${ }^{19}$ and Elhadidi et al. $^{20}$, had found lower prevalence of hypothyroidism $6 \%, 4.4 \%, 3.9 \%, 4.3 \%, 5.7 \%$ \& $4 \%$ respectively. And this difference could be explained 
by the smaller patient numbers, difference in sensitivity of enzyme linked immunosorbent assay (ELISA) used to measure TSH and may be difference of prevalence of hypothyroidism in different countries.

Our study found that the the most common thyroid dysfunction among SLE patients was elevated TSH as it was found in $27(45 \%)$ of all SLE patients [18 $(60 \%)$ patients in group I (active SLE) and 9 (30\%) patients in group II (inactive SLE)] and the next common dysfunction was low free $\mathrm{T} 4$ as it was found in $22(36.66 \%)$ of all SLE patients [18 $(60 \%)$ patients in group I (active SLE) versus $4(13.33 \%)$ patients in group II (inactive SLE)]. Our results was in concordant with Miller et al. ${ }^{8}$, who found that more than $45 \%$ of these patients had elevated levels of TSH, $34 \%$ had low T3 determinations. Also Magaro et al. ${ }^{21}$, reported that The SLE patients had significantly lower T4 levels than the controls. Zakeri. \& Sandooghi ${ }^{12}$ reported that elevated TSH levels were the most common dysfunction in SLE patients which agreed with our results however with lesser incidence $19.3 \%$ and low TSH was the next most common dysfunction in $4.8 \%$ of patients rather than low $\mathrm{T} 4$ in our results.

In this study the thyroid antibodies were present in patients in $13(21.67 \%)$ SLE patients [12 (40\%) patients were in group I (active SLE) and 1 (3.33\%) patient was in group II (inactive SLE)] while non were present in control group. $11(18.33 \%)$ patients had Anti-TG and $2(3.33 \%)$ had Anti-TPO. The presence of autoantibodies suggests autoimmunity to be the basis for thyroid dysfunction in SLE patients and it proved by more predominance of antibodies in active SLE patients. Our results were lower than Weetman \& Walport ${ }^{9}$, who reported that the prevalence of thyroid antibodies was $51 \%$. Also Magaro et al. ${ }^{21}$, found that high prevalence of antithyroid antibodies has been found in patients with SLE. $45.5 \%$ of patients with active SLE presented antithyroid antibodies and anti TG and anti TPO antibodies were not found in patients with inactive SLE nor in controls. Also Kumar et al. ${ }^{14}$, found that euthyroid state with the elevation of antibodies alone was seen in $12 \%$ of the lupus patients and the prevalence of thyroid autoantibodies in lupus patients was $30 \%$. Also our results were lower than Miller et al. ${ }^{8}$, who had found that $18 \%$ of his patients had high anti TPO antibody titers. Chan et al. ${ }^{10}$, has shown that positive anti TPO were detected in $23.2 \%$ of SLE patients. Pyne \& Isenberg ${ }^{19}$ also found that the frequency of thyroid antibodies $14 \%$ which were anti TPO and anti TG antibodies. In Egypt, El-Sharif et al. ${ }^{11}$, revealed that TPO antibody was found in $15 \%$ of SLE patients. Also, Zakeri. \& Sandooghi ${ }^{12}$ reported higher positive TPO antibody was detected in $16.9 \%$ of SLE and $16.3 \%$ of the control group.

Our study found that the females were likely to have thyroid dysfunction than males. This result was agreed with Porkodi et al. ${ }^{16}$, who found all SLE patients with thyroid dysfunction were females. Also Antonelli et al. ${ }^{13}$, found that any kind of thyroid disorders and thyroid antibodies had a higher prevalence in women than in men. Also, Kumar et al. ${ }^{14}$, reported that all his studied SLE patients with thyroid dysfunction were women.

In our study SLE patients were subdivided according to thyroid function. Our results showed that there was no statistical significant difference between three groups (hypothyroidism group, subclinical hypothyroidism group, \& euthyroid group) as regard age of patients. But this didn't agree with Porkodi et al. ${ }^{15}$, who reported that clinical hypothyroid was belonged to an older age group (mean age 30.4 years), Subclinical hypothyroid (TSH alone raised) was in (mean age 28 years) and hyperthyroidism occurred in younger individuals (mean age 27 years). Also Weetman \& Walport ${ }^{9}$, Vianna et al. ${ }^{16}$ and Park et al. ${ }^{22}$ studies also reported that thyroid dysfunction more common in older age. But, Parente et al. study ${ }^{23}$ had found that in SLE schoolchildren patients had higher hypothyroid function tests.

Our study showed that no statistical significant difference between 3 groups as regard disease duration and this result was in agreement with with Kumar et al. ${ }^{14}$, who reported that SLE disease duration had no statistically significant association with thyroid dysfunction, however Weetman \& Walport ${ }^{9}$, Vianna et al. ${ }^{16}$, and Park et al. ${ }^{22}$, reported that thyroid dysfunction more common in longer duration of SLE disease.

Our results showed that there was highly significant difference between the 3 groups as regard SLAM score; and this is confirmed by significantly higher ESR \& serum creatinine in hypothyroid group than subclinical hypothyroidism group than the euthyroid group. This was in agreement with Magaro et al. ${ }^{21}$, who reported that the thyroid function appears to be dependent on the activity of the systemic autoimmune process. Also our results were in agreement with Kumar et al. ${ }^{14}$, who also reported that SLEDAI score when compared between lupus patients with thyroid dysfunction to those with normal thyroid function. A statistically significant association was found between SLEDAI and thyroid dysfunction. But our results were disagreed with Hrycek et al. ${ }^{24}$, who reported that fT3 and fT4 concentrations are lower in SLE treated women with mild disease activity.

Our result showed that the patients with thyroid dysfunction had complaining more from non-specific symptoms. In their study Magaro et al. ${ }^{10}$, reported that mild hypothyroidism in SLE is clinically silent. Also, Zakeri. \& Sandooghi ${ }^{12}$ reported that the absence of clinical thyroid symptoms in their patients. Also Allahabadia et al. $^{25}$, reported that the symptoms and signs of hypothyroidism are often subtle and vague. 
They are not specific which means they can mimic the symptoms of many other conditions and are often attributed to aging in their SLE patients.

In our study we found that there was statistically significant positive correlation between $\mathrm{Ab}$ TG and ESR, BMI \& SLAM score and significantly negative correlation with Free T3 and T4. Till now there is no available studies explain the association between increasing the SLE disease activity scores and increasing the thyroid antibodies. Our data agrees with Parente et al. ${ }^{23}$, who found that higher levels of thyroid antibodies as well as hypothyroid function tests in his SLE schoolchildren patients with higher disease activity assessed by Systemic Lupus Erythematosus Disease Activity Index (SLEDAI).

There was no significant difference between 3 groups as regard U/S finding. The overall incidence of U/S abnormality among SLE patients 4 (6.67\%) patients had thyroid cyst, $2(3.33 \%)$ had multinodular goiter \& $1(1.67 \%)$ patient had surgically resected left thyroid lobe. Few papers in literature discussed thyroid $\mathrm{U} / \mathrm{S}$ in SLE patients. Antonelli et al. ${ }^{13}$, reported that there was no significant difference between the SLE patients \& control group as regard hypoechoic pattern \& thyroid volume among males but there was significant difference between the 2 groups among females as SLE female patients had higher percentage of hypoechoic pattern $29.8 \%$ versus $13.1 \%$ in control group and less thyroid volume $12 \%$ versus $3.3 \%$ in control group. Also they found that the SLE patients with high TSH had more hypoechoic pattern \& larger thyroid volume than the SLE patients with low TSH. Quintanilla-Flores et al. ${ }^{26}$, had found that the prevalence of thyroid nodule (TN) was $27 \%$ in 55 SLE patients > 16 years of age. One-third of TN were $>1 \mathrm{~cm}$ with radiological features of malignancy. They also reported that the mean age of patients with TN was $39 \pm 11$ years, $93 \%$ was women with SLE duration $10 \pm 6$ years.

In conclusion; thyroid dysfunction is more frequent in SLE patients than control group. Subclinical and overt hypothyroidism are more likely to occur in SLE patients than hyperthyroidism. There is a positive correlation between $\mathrm{Ab}$ TG level and disease activity however the correlation with anti TPO was non-significant, this might suggest that in Egyptian patients anti-TG may be a better marker to predict hypothyroidism in these patients. The higher frequency of thyroid dysfunction and higher levels of thyroid antibodies in active SLE patients rather than inactive one might suggest the role of presence of hypothyroidism in increasing the disease activity in SLE patients and whether treatment of such condition improves the SLE disease activity or not; this issue needs to be more investigated.

[Disclosure: Authors report no conflict of interest]

\section{REFERENCES}

1. Rojas-Villarraga A., Toro C., Espinosa G., Rodríguez-Velosa Y., Duarte-Rey C., Mantilla R D., Iglesias-Gamarra A., Cervera R. \& Anaya J-M.: "Factors influencing polyautoimmunity in systemic lupus erythematosus." Autoimmunity Reviews. 2010; 9: 229-32.

2. Griffiths B.: "Assessment of patients with systemic lupus erythematosus and the use of lupus disease activity indices." Best Practice \& Research Clinical Rheumatology 2005; 19: 685-708.

3. Scofield RH. "Autoimmune thyroid disease in systemic lupus erythematosus and Sjögren's syndrome". Clin Exp Rheumatol 1996; 14: 321-30.

4. Zakeri Z. \& Sandooghi M. "Thyroid Disorder in Systemic Lupus Erythematosus Patients in Southeast Iran." Shiraz E Medical Journal.2010; 11: 34-8.

5. Hochberg MC. Updating the American College of Rheumatology revised criteria for the classification of Systemic Lupus Erythematosus. Arthritis Rheum. 1997; 40:1725.

6. Liang MH., Socher A., Larson MG., Schur PH. Reliability and validity of six systems for the clinical assessment of disease activity in SLE. Arthritis Rheum.1989; 32:1107-18.

7. Courtney PA, Crockard AD, Williamson K, Irvine AE, Kennedy RJ, Bell AL. Increased apoptotic peripheral blood neutrophils in systemic Lupus Erythematosus: relations with disease activity, antibodies to double stranded DNA, and neutropenia. Ann Rheum Dis.1999: 58: 309-14.

8. Miller FW., Moore GF., Weintraub BD. \& Steinberg AD. "Prevalence of thyroid disease and abnormal thyroid function test results in patients with systemic lupus erythematosus." Arthritis Rheum.1987; 30: 1124-31.

9. Weetman AP. \& Walport MJ. "The association of autoimmune thyroiditis with systemic lupus erythematosus." Br J Rheumatol.1987; 26: 359-61.

10. Chan AT., Al-Saffar Z. \& Bucknall RC. "Thyroid disease in systemic lupus erythematosus and rheumatoid arthritis." Rheumatology.2001; 40: 353-4.

11. El-Sherif WT., El Gendi SS., Ashmawy MM ., Ahmed HM . \& Salama MM. "Thyroid disorders and auto antibodies in systemic lupus erythromatosus and rheumatoid arthritis." Egypt $\mathbf{J}$ Immunol.2004; 11: 81-90.

12. Zakeri Z. \& Sandooghi M. "Thyroid Disorder in Systemic Lupus Erythematosus Patients in Southeast Iran." Shiraz E Medical Journal.2010; 11: $34-8$.

13. Antonelli A., Fallahi P., Mosca M., Ferrari SM., Ruffilli I.,Corti A., Panicucci E., Neri R. \& Bombardieri S. " Prevalence of thyroid dysfunctions in systemic lupus erythematosus." Metabolism Clinical and Experimental.2010; 59: 896-900. 
14. Kumar K., Kole AK., Karmakar PS. \& Ghosh A. "The spectrum of thyroid disorders in systemic lupus erythematosus." Rheumatol Int. 2012; 32: 73 8.

15. Porkodi R., Ramesh S., Mahesh A., Kanakarani P., Rukmangathrajan S. \& Rajendran PC. "Thyroid dysfunction in systemic lupus erythematosus and rheumatoid arthritis." J Indian Rheumatol Assoc. $2004: 12: 0-0$.

16. Vianna JL., Haga HJ., Asherson RA., Swana G. \& Hughes GRV. "A prospective evaluation of antithyroid antibody prevalence in 100 patients with systemic lupus erythematosus. J Rheumatol 1991; 18:1193-5.

17. Tsai RT., Chang TC., Wang CR., Chuang CY. \& Chen CY. "Thyroid disorders in Chinese patients with systemic lupus erythematosus." Rheumatol Int.1993; 13: 9-13.

18. Boey ML., Fong PH., Lee JSC., Ng WY. \& Thai AC. "Autoimmune thyroid disease in SLE in Singapore." Lupus 1993; 2: 51-4.

19. Pyne D. \& Isenberg DA. "Autoimmune thyroid disease in systemic lupus Erythematosus." Ann Rheum Dis.2002; 61:70-2.

20. Elhadidi KT., Mansour MA., Elwakd MM. \& Elemary AM. "Thyroid dusfunction and thyroid antibodies in Egyptian patients with SLE and its impact on musculoskeletal manifestations of the disease." Thesis rheumatology \& rehabilitation 2012, Cairo University.

21. Magaro M., Zoli A., Altomonte L., Mirone L., La Sala L., Barini A. \& Scuderi F "The association of silent thyroiditis with active systemic lupus erythematosus." Clin Exp Rheumatol.1992;10:67.

22. Park DJ., Cho C., Lee Sh. \& Kim HY. "Thyroid disorders in Korean patients with systemic lupus erythematosus." Scand J Rheumatol. 1995; 24:13-7.

23. Parente CL., Bonfá E., Martinago CD., de Oliveira RM., Carvalho JF. \& Pereira RM. "Juvenile onset Systemic Lupus Erythematosus thyroid dysfunction: a subgroup with mild disease?" J Autoimmun.2009; 33: 121-4.

24. Hrycek A., Hrycek E., Kopczyński P., Kopczyńska M., Romanowski Ł. \& Walawska A. "The thyroid functional tests, antithyroid antibodies and the level of prolactin in treated patients with systemic lupus erythematosus". Pol Merkur Lekarski.2010; 29: 231-4.

25. Allahabadia A., Razvi S., Abraham P.\& Franklyn J. "Diagnosis and treatment of primary hypothyroidism." BMJ.2009; 338: b725.

26. Quintanilla-Flores DL., Hernández-Coria MI., Elizondo-Riojas G., Galarza-Delgado DA., González-González JG. \& Tamez-Pérez HE. "Thyroid nodules in Hispanic patients with systemic lupus erythematosus." Lupus.2013. 